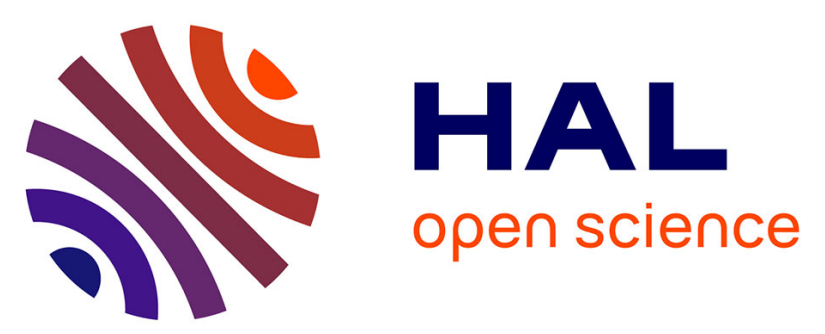

\title{
Generalized Boolean logic Driven Markov Processes: a powerful modeling framework for Model-Based Safety Analysis of dynamic repairable and reconfigurable systems
}

Pierre-Yves Piriou, Jean-Marc Faure, Jean-Jacques Lesage

\section{To cite this version:}

Pierre-Yves Piriou, Jean-Marc Faure, Jean-Jacques Lesage. Generalized Boolean logic Driven Markov Processes: a powerful modeling framework for Model-Based Safety Analysis of dynamic repairable and reconfigurable systems. 2016. hal-01393700

\author{
HAL Id: hal-01393700 \\ https://hal.science/hal-01393700
}

Preprint submitted on 8 Nov 2016

HAL is a multi-disciplinary open access archive for the deposit and dissemination of scientific research documents, whether they are published or not. The documents may come from teaching and research institutions in France or abroad, or from public or private research centers.
L'archive ouverte pluridisciplinaire HAL, est destinée au dépôt et à la diffusion de documents scientifiques de niveau recherche, publiés ou non, émanant des établissements d'enseignement et de recherche français ou étrangers, des laboratoires publics ou privés. 


\title{
Generalized Boolean logic Driven Markov Processes: a powerful modeling framework for Model-Based Safety Analysis of dynamic repairable and reconfigurable systems
}

\author{
Pierre-Yves Piriou $^{\mathrm{a}}$, Jean-Marc Faure ${ }^{\mathrm{b}}$, Jean-Jacques Lesage ${ }^{\mathrm{c}}$ \\ ${ }^{a}$ Electricité de France, REDD, 78400 Chatou, France \\ ${ }^{b}$ LURPA, ENS Cachan, Univ. Paris Sud, Supmeca, Univ. Paris-Saclay, 94235 Cachan, France \\ ${ }^{c}$ LURPA, ENS Cachan, Univ. Paris Sud, Univ. Paris-Saclay, 94235 Cachan, France
}

\begin{abstract}
This paper presents a modeling framework that permits to describe in an integrated manner the structure of the critical system to analyze, by using an enriched fault tree, the dysfunctional behavior of its components, by means of Markov processes, and the reconfiguration strategies that have been planned to ensure safety and availability, with Moore machines. This framework has been developed from BDMP (Boolean logic Driven Markov Processes), a previous framework for dynamic repairable systems. First, the contribution is motivated by pinpointing the limitations of BDMP to model complex reconfiguration strategies and the failures of the control of these strategies. The syntax and semantics of GBDMP (Generalized Boolean logic Driven Markov Processes) are then formally defined; in particular, an algorithm to analyze the dynamic behavior of a GBDMP model is developed. The modeling capabilities of this framework are illustrated on three representative examples. Last, qualitative and quantitative analysis of GDBMP models highlight the benefits of the approach.
\end{abstract}

Keywords: Model Based Safety Analysis, Generalized Boolean logic Driven Markov Processes, Dynamic and repairable system, Reconfiguration strategies, Moore machine

\section{Introduction}

Safety analysis of a critical system requires first that the structure of this system has been previously modeled. Qualitative and quantitative analysis results depend indeed not only on the features of the system components but also on their organization (serial or parallel configuration, $k$-out-of- $n$ redundancies). System structure is classically modeled by a tree with logical gates in fault tree analysis, a popular and widespread safety assessment technique in industry. A weakness of this approach has been identified since more than twenty years, however. Only combinations of faults are considered whereas in some cases the failure of the system depends on fault sequences. This explains why several proposals of dynamic ([1]), or temporal ([2]), fault trees that permit to obtain these sequences have been published; formalization of the dynamic gates that are included in

Email addresses: pierre-yves .piriou@edf .fr (Pierre-Yves Piriou), jean-marc.faure@ens-cachan.fr (Jean-Marc Faure), jean-jacques. lesage@ens-cachan.fr (Jean-Jacques Lesage) these trees by means of Petri nets ([3]), Markov chains ([4]), algebraic approaches ([5], [6] [7], [8], [9] and [10]), has been also presented.

All these extensions of the original fault-tree method have assumed that the components of the system under analysis are not repairable, which is not the case for every critical system and in particular for systems whose duration of the mission is over several years, like power plants and power distribution networks. New modeling frameworks (e.g. [11], [12], [13] and [14]) have then been developed. These formalisms allow to model explicitly, in addition to the structure of the system, the dysfunctional behavior of its components by using for instance Markov processes or transition systems.

Nevertheless, despite the benefit of these worthwhile contributions for a more accurate safety analysis, an issue remains. Redundancies management requires to define reconfiguration strategies, e.g. to describe how the service is transferred from a main component which has failed to one or several spare components and how the operation of the main component is resumed once it has 
been repaired. Reconfiguration strategies can be com- 89 plex when multi-state components are considered and deserve to be explicitly and formally described. Moreover, they are performed by human operators or, more and more frequently, automatic systems. Whatever the nature of this reconfiguration controller, it may fail and this failure can impact safety ([15]). Hence, a reconfiguration strategy may fail either because the coverage of the fault(s) that trigger(s) this reconfiguration is not perfect or because its control fails. Numerous worthwhile results ([16] and [17] for instance) have been previously obtained to deal with the first issue. The aim of this paper is to tackle out the second issue.

Therefore we propose a novel modeling framework that supports Model Based Safety Analysis (MBSA) of dynamic repairable and reconfigurable systems. It per- 101 mits to describe at once the structure of the critical sys- 102 tem with a causal tree, the dysfunctional behavior of its 103 components by means of switched Markov processes, 104 and the reconfiguration strategies with Moore machines. It has been termed Generalized Boolean logic Driven Markov Processes (GBDMP) because it generalizes the BDMP frame defined in ([11]). A draft version of this framework has been sketched in ([18]); only modeling of reconfiguration strategies in a non-formal manner was considered in this reference. The current paper presents a widely extended - modeling of the structure of the system and of the dysfunctional behavior of components is now also considered - and far more formal- 112 ized version.

The outline of the paper is the following. Section $2{ }^{113}$ starts with a reminder on BDMP; the limitations of this 114 framework for reconfiguration modeling are then shown 115 in this section. The syntax and semantics of GBDMP are detailed respectively in the third and the fourth section; the evolution rules of a GBDMP model are stated and an algorithm to animate such a model according to these rules is proposed too. This theoretical contribution 119 is illustrated in the fifth section with three simple but ${ }_{120}$ representative examples whereas section 6 focuses on qualitative and quantitative analysis of GBDMP models. Finally, concluding remarks and perspectives are ${ }^{122}$ drawn up in section 7 .

\section{Modeling with BDMP}

The BDMP framework has been introduced ([11]) for safety analysis of systems whose components are re- ${ }_{127}$ pairable. To meet this objective, the structure is mod- 128 eled by a fault tree that includes not only logical gates 129 but also triggers; the role of a trigger is to require or 130 not some nodes of the tree. Moreover, the leaves of ${ }_{131}$ the tree are no more basic events which can be represented by Boolean variables but a description of the failure/repair behavior of components in the form of Triggered Markov Processes (TMP). The formal definition of BDMP is reminded and exemplified below; discussion of the example permits to pinpoint the limitations of BDMP for reconfiguration modeling.

\subsection{Formal definition}

Definition 1. Formally, a BDMP is a 4-tuple $<\mathcal{F}$, te $, T,\left(P_{i}\right)>[11]$ where:

- $\mathcal{F}$ is a multi-top fault tree, i.e. a 3-tuple $\langle N, E, \kappa>$ where:

- $N=G \cup L$ is a set of nodes, which is partitioned in two disjoint sets: $G$ (set of gates) and L (set of leaves);

- $E \subseteq G \times N$ is a set of oriented edges, such that $\langle N, E\rangle$ is a directed acyclic graph;

- $\kappa \in G \longrightarrow \mathbb{N}^{*}$ is a function that determines the gates kind. Let $g$ be a gate which has $n$ sons: if $\kappa(g)=n$ then $g$ is an AND gate, if $\kappa(g)=1$ then $g$ is an OR gate, and more generally, if $\kappa(g)=k$ then $g$ is a $k / n$ gate;

- te $\in G$ is the top event of $\mathcal{F}$;

- $T \subseteq(N \backslash\{t e\}) \times(N \backslash\{t e\})$ is a set of triggers;

- $P$ is a set of Triggered Markov Processes (TMP) associated to the leaves. A TMP is a 5-tuple $<\mathcal{Z}_{0}, \mathcal{Z}_{1}, \mathcal{X}_{F}, f_{0 \rightarrow 1}, f_{1 \rightarrow 0}>$ where:

- $\mathcal{Z}_{0}$ and $\mathcal{Z}_{1}$ are two homogeneous continuous Markov chains. We denote by $\mathcal{X}_{0}$ and $\mathcal{X}_{1}$ their respective state spaces;

- $\mathcal{X}_{F} \subseteq \mathcal{X}_{0} \cup \mathcal{X}_{1}$ is the subset of failure states;

- $f_{0 \rightarrow 1} \in \mathcal{X}_{0} \times \mathcal{X}_{1} \longrightarrow[0,1]$ is the probabilistic transfer function between $X_{0}$ and $X_{1}$;

- $f_{1 \rightarrow 0} \in X_{1} \times X_{0} \longrightarrow[0,1]$ is the probabilistic transfer function between $X_{1}$ and $X_{0}$.

\subsection{Example of $B D M P$}

A BDMP model is depicted in Figure 1. The set of gates in the fault tree is $G=\{G 1, G 2, G 3\}$ with $\kappa(G 1)=2 ; \kappa(G 2)=\kappa(G 3)=1$. The set of leaves is $L=\{C 1, C 2, C 3\}$. One trigger is introduced (dashed arrow from $G 2$ to $G 3$ ); this trigger means that when the output of $G 2$ is True (resp. False) the part of the system related to $G 3$ ( $C 2$ or $C 3$ ) is required (resp. not required). 


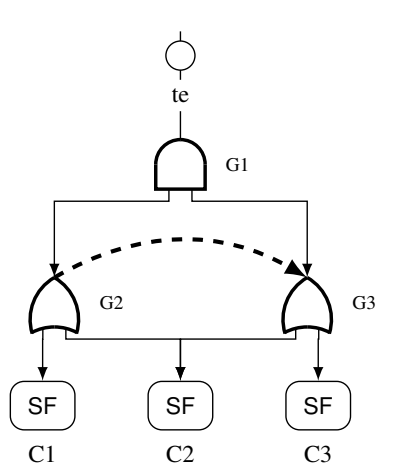

a)
SF

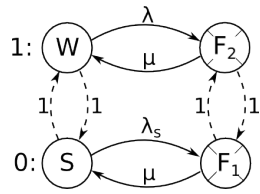

b)

Figure 1: Example of BDMP. a) Fault tree modeling the structure; b) 169

TMP associated to every leaf

The TMP associated to every leaf ${ }^{1}$ comprises four ${ }_{173}$ states: $S$ (Standby), $F 1$ (Faulty during standby), $W{ }_{174}$ (Working) and F2 (Faulty during working). The 175 solid arrows represent transitions of continuous Markov chains (the label of the transition is a failure or repair 176 rate in this case), whereas the dashed arrows represent 177 operation mode changes, from standby to working and 178 vice versa (the label of the transition is then the proba- 179 bility of firing when the change is required). Thus, this 180 TMP is composed of two Markov chains which describe 181 the behavior of a working and standby component and ${ }_{182}$ are connected by two transfer functions which model 183 the actions of the trigger.

To sum up, a BDMP model is a fault tree whose leaves are TMP. The state of each node $n$ (leaf or gate) ${ }^{185}$ is characterized by two Boolean variables that represent ${ }^{186}$ its activation status $M_{n}$ and its failure status $F_{n}$. The ${ }^{187}$ activation statuses are controlled by the triggers; when ${ }^{188}$ the origin of a trigger is faulty (respectively not faulty), ${ }^{189}$ the destination is required (respectively not required). ${ }^{190}$ Hence, a node is activated ( $M_{n}$ becomes True) if and ${ }^{191}$ only if it is required and at least one of its fathers in the ${ }^{192}$ tree is activated, assuming that the top event is always active. The failure status of a gate is computed from the failure statuses of its sons like in classical fault tree analysis.

\footnotetext{
${ }^{1}$ It is possible to associate different types of TMP, with always two Markov chains, to the leaves [11]. The transition from $S$ to $F 1$ is removed if it is assumed that the component cannot fail in standby mode, for instance. Furthermore, failure on-demand can be easily modeled by replacing the transition from $S$ to $W$ by two transitions: one from $S$ to $F 1$ with a probability $p$ (failure probability) and the other one from $S$ to $W$ with a probability $1-p$. Nevertheless, only 200 one type will be considered here for brevity reasons.
}

\subsection{Reconfiguration modeling}

The concept of trigger that is introduced by the BDMP framework is a first attempt to model reconfiguration. Despite its novelty and interest when repairable systems are considered, this modeling primitive presents three limitations:

- First, only one reconfiguration strategy is considered: the destination of the trigger is activated as soon as the origin of the trigger fails and is deactived as soon as the origin is repaired. This strategy is not the only one which is used in practice, however. When standby redundancy is implemented with two identical components, with the same failure rate, for instance, it is frequent to activate the origin, once repaired, only when the destination has failed to balance the working durations of the two components, and decrease the risk of failure on demand, if it exists.

- Second, the models of components (leaves of the fault tree) include only two operation modes: working and standby. Nonetheless, real components of critical systems may have more than two modes, for instance a standby mode, a normal mode and an overspeed mode, the latter one being a solution to perform the service during a limited time when the component is the only faultless one that remains.

- Last, possible failure of the trigger is not considered. It is assumed indeed that, when the origin of a trigger fails, the trigger always sends to its destination a request to move to the working operation mode. This is unfortunately not always true in practice, and especially when the trigger is implemented by an automatic system that comprises electronic boards, relays, etc. which may fail.

To overcome these limitations (restricted number of reconfiguration strategies, of operation modes, failure of the control of the reconfiguration not considered) a novel framework is defined in the next section.

\section{Generalized Boolean logic Driven Markov Pro- cesses (GBDMP)}

GBDMP have been defined from BDMP by replacing first the concept of trigger by that of switch whose behavior is described by a Moore machine; complex 
reconfiguration strategies can then be modeled. Moreover, TMP are replaced by SMP (Switched Markov Processes) to model components with more than two operation modes. Last, control of the reconfiguration strategies is explicitly modeled and connected to switches; hence, the impact of failures of this control can be considered.

The syntax of these models is first detailed in what follows; properties that must be satisfied by wellformed GBDMP are stated too.

\subsection{Overall description}

Definition 2. A Generalized Boolean logic Driven Markov Processes is a 6-tuple $\langle V, E, \kappa, v, s t r, s m p>$ where ${ }^{2}$ :

- $V=N \cup S=G \cup L \cup S$ is a set of vertices partitioned into the nodes (i.e. the gates and the leaves) and the switches.

- $E=E_{F} \cup E_{S}$ is a set of oriented edges, such that $E_{F} \subseteq G \times N$ and $E_{S} \subseteq(N \times S) \cup(S \times N)$;

- $\kappa: G \rightarrow \mathbb{N}^{*}$ is a function that determines the gates kind (just as with BDMP);

- $v: E \rightarrow \mathbb{N}$ is a function that associates an integer ${ }_{245}$ label to each edge;

- $s t r: S \rightarrow \mathbb{M}$ is a function that associates a Moore ${ }^{247}$ machine (a strategy) to each switch;

- smp :C $\rightarrow \mathbb{P}$ is a function that associates a SMP ${ }^{250}$ to each component.

A simple GBDMP is shown at Figure 2. The graphi- ${ }^{252}$ cal representation of leaves and gates of the fault tree is ${ }^{253}$ the same as for BDMP. A dashed rectangle represents a ${ }^{254}$ switch $(S=\{S 1\})$ and the solid (resp. dashed) arrows ${ }^{255}$ the edges of $E_{F}$ (resp. $E_{S}$ ), which connect respectively ${ }_{256}$ the gates to the nodes (leaves or gates) and the switches ${ }_{257}$ to the nodes or the nodes to the switches; the label of an edge is the value of the function $v$ for this edge. The ${ }^{258}$ behavior of the leaves $C 1, C 2, C 3$ and $C 4$ is depicted at ${ }^{259}$ part b) of Figure 2 and that of $S 1$ at part c). Compared to 260 Figure 1, this GBDMP includes a new component $(C 4){ }_{261}$ that is in charge of reconfiguration.

Two directed graphs can be defined in the structure of ${ }^{262}$ a GBDMP model:

${ }^{2} \mathbb{M}$ and $\mathbb{P}$ designate respectively the set of Moore machines and ${ }^{265}$ the set of SMP.

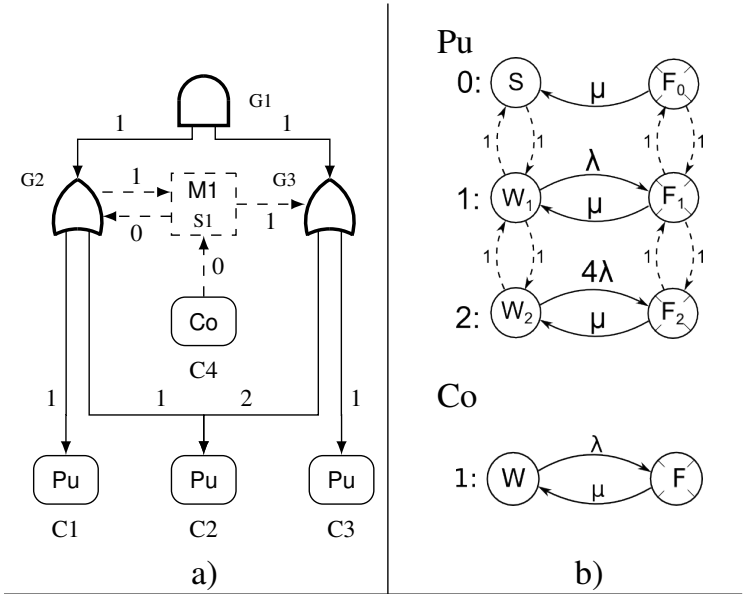

M1

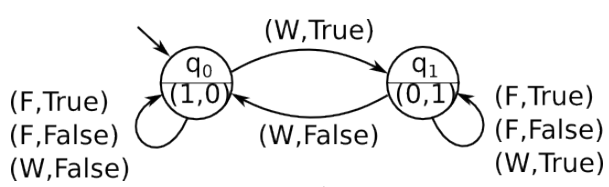

c)

Figure 2: Example of GBDMP. a) Structure modeling; b) SMP Pu (associated to $C 1, C 2, C 3$ ) and $C o$ (associated to $C 4$ ); c) Moore machine $M 1$ (associated to $S$ 1)

- $\mathcal{G}_{F}=<N, E_{F}>$ is the graph classically called the Fault Tree. The label of an edge of this graph corresponds to an operation mode of the destination node. At figure $2 \mathrm{a}$ ), for instance, the labels of the two edges from $G 3$ to $C 2$ and $C 3$ mean that these leaves must be respectively in their second and first operation mode when this gate is required. In a similar way, $C 2$ must be in its first (resp. second) operation mode when $G 2$ (resp. G3) is required.

- $\mathcal{G}_{S}=<V, E_{S}>$ is the graph where switches are connected to nodes. The labels of edges of this graph correspond merely to numbers of the inputs and outputs of switches.

For every vertex of these two graphs, it is possible to define:

- the sets of its downstream and upstream vertices:

$\forall n \in N, \Gamma_{\mathcal{G}_{F}}^{-}(n)=\left\{g \in G \mid(g, n) \in E_{F}\right\}$

$\forall g \in G, \Gamma_{\mathcal{G}_{F}}^{+}(g)=\left\{n \in N \mid(g, n) \in E_{F}\right\}$

$\forall s \in S, \Gamma_{\mathcal{G}_{s}}^{-}(s)=\left\{n \in N \mid(n, s) \in E_{S}\right\}$

$\forall s \in S, \Gamma_{\mathcal{G}_{S}}^{+}(s)=\left\{n \in N \mid(s, n) \in E_{S}\right\}$

- its indegree and outdegree ( $\mathcal{G}$ can be replaced by $\mathcal{G}_{F}$ or $\mathcal{G}_{S}$ herebelow):

$\forall v \in V, d_{\mathcal{G}}^{-}(v)=\operatorname{Card}\left(\Gamma_{\mathcal{G}}^{-}(v)\right)$

$\forall v \in V, d_{\mathcal{G}}^{+}(v)=\operatorname{Card}\left(\Gamma_{\mathcal{G}}^{+}(v)\right)$ 
These notations will be used in the remainder of this ${ }_{314}$ section, in particular to state the consistency properties 315 of well-formed GBDMP.

\subsection{Leaf behavior modeling}

The behavior of a leaf is modeled by a $k$-SMP which ${ }^{318}$ is composed of $k$ Markov chains. Each Markov chain ${ }^{319}$ corresponds to an operation mode and comprises fault- 320 less and faulty states; the transitions between these states are stochastic because they model mainly failures and repairs. It must be noted that the Markov chains that compose a k-SMP are not necessarily homogeneous; different distributions (e.g. exponential, lognormal, Weibull) can be associated to transitions. However, quantitative analysis of the constructed model requires the tool which will be selected for this analysis is able to deal with the distributions introduced in the model. In the examples of this paper, only exponential distributions (then constant failure and repair rates) will 329 be considered because this distribution is the most com- 330 mon one.

In the example of Figure $2 \mathrm{~b}$ ), the 3-SMP associated to the leaves $C 1, C 2$ and $C 3$ comprises three Markov chains to represent a component with two working modes and one standby mode; in this model, it ${ }_{334}$ is assumed that no failure occurs in the standby mode ${ }_{335}$ and that the failure rate in the second working mode is 336 greater than the corresponding rate in the first working mode. The set of states $X^{P}$ of the $k$-SMP $P$ is the union ${ }^{337}$ of the sets of states of the chains; similarly, the set of 338 states $\mathcal{X}_{F}^{P}$ of the k-SMP is the union of the sets of faulty ${ }^{339}$ states of the chains. $k(k-1)$ probabilistic transfer functions between the chains of a $k$-SMP must be defined. The value of the transfer function between two states of two different chains is equal to 1 (deterministic transfer) if no failure on-demand is considered (case of Figure 2 b) when the operation mode is changed and belongs to $[0,1]$ otherwise.

Definition 3. A k-mode Switched Markov Process (k$S M P)$ is defined as a 3-tuple $P=<\left(\mathcal{Z}_{i}^{P}\right)_{0 \leq i<k}, \mathcal{X}_{F}^{P},\left(f_{i \rightarrow j}^{P}\right)_{(i, j) \in \llbracket 0, k-1 \rrbracket^{2}}>$ where:

- $\left(\mathcal{Z}_{i}^{P}\right)_{0 \leq i<k}$ is a family of Markov chains i.e. $\forall i \in$ $\llbracket 0, k-1 \rrbracket, \mathcal{Z}_{i}^{P}$ is a 3-tuple $<\mathcal{X}_{i}^{P}, A_{i}^{P}, p 0_{i}^{P}>$ where:

- $X_{i}^{P}$ is a finite set of states;

- $A_{i}^{P}:\left(\mathcal{X}_{i}^{P}\right)^{2} \rightarrow \mathbb{R}^{+}$is the matrix of transition ${ }^{342}$ rates;

- $p 0_{i}^{P}: \mathcal{X}_{i}^{P} \rightarrow[0,1]$ is the initial probability distribution $\left(\sum_{x \in \mathcal{X}_{i}^{P}} p 0_{i}^{P}(x)=1\right)$;
$\left(X^{P}=\bigcup_{i=0}^{k-1} X_{i}^{P}\right.$ denotes the set of all states of the $S M P)$

- $\mathcal{X}_{F}^{P} \subseteq \mathcal{X}^{P}$ is the subset of failure states;

- $\left(f_{i \rightarrow j}^{P}\right)_{(i, j) \in \llbracket 0, k-1 \rrbracket^{2}}$ is a family of probabilistic transfer functions, i.e.

$\forall(i, j) \in \llbracket 0, k-1 \rrbracket^{2}, f_{i \rightarrow j}^{P}: X_{i}^{P} \times X_{j}^{P} \longrightarrow[0,1]$ such that $\forall x \in \mathcal{X}_{i}^{P}, \sum_{y \in \mathcal{X}_{j}^{P}} f_{i \rightarrow j}^{P}(x, y)=1$.

When a k-SMP is associated to a leaf, it is said that the dimension of this leaf is equal to $\mathrm{k}$. The activation status of a leaf whose dimension is greater than 2 cannot be represented by a Boolean variable, as this was the case with $\mathrm{BDMP}^{3}$, but by an integer. Calculus of the value of this integer variable will be dealt with in the next subsection.

\subsection{Node status variables}

For each node (leaf or gate) $n \in N$ of the fault tree, three status variables must be defined:

- $F_{n}$ : a Boolean variable $\left(F_{n} \in\{\right.$ False, True $\left.\}\right)$ that represents the failure status of the node $\left(F_{n}=\right.$ True $\Leftrightarrow n$ is faulty);

- $R_{n}$ : a binary variable $\left(R_{n} \in\{0,1\}\right)$ that represents the requirement status of the node $\left(R_{n}=1 \Leftrightarrow n\right.$ is required to perform the service);

- $M_{n}$ : a positive integer variable $\left(M_{n} \in \mathbb{N}\right)$ that represents the activation status of the node $\left(M_{n}=k \Leftrightarrow n\right.$ is in the operation mode number $k$ ).

The failure statuses are determined as follows:

- For a leaf $l \in L, F_{l}$ is True when the active state of the SMP associated to this leaf (denoted $X_{l}$ ) is a faulty state.

$$
X_{l} \in \mathcal{X}_{F}^{s m p(l)} \Rightarrow F_{l}=\text { True }
$$

- For a gate $g \in G, F_{g}$ is True when the number of its sons that are either faulty or non-required is greater than $\kappa(g)$.

$$
\operatorname{Card}\left(\left\{n \in \Gamma_{\mathcal{G}_{F}}^{+}(g) \mid F_{n} \vee \neg R_{n}\right\}\right) \geq \kappa(g) \Rightarrow F_{g}=\text { True }
$$

In the example of Figure 2, the failure statuses of the leaf $C 1$ and the gates $G 1$ and $G 2$, for instance, are respectively obtained as follows:

\footnotetext{
${ }^{3} \mathrm{~A}$ TMP can be seen as a 2-SMP.
} 
- $X_{C 1} \in\left\{F_{0}, F_{1}, F_{2}\right\} \Rightarrow F_{C 1}=$ True

- $\operatorname{Card}\left(\left\{n \in\{G 2, G 3\} \mid F_{n} \vee \neg R_{n}\right\}\right) \geq 2 \Rightarrow F_{G 1}=$ True

- $\operatorname{Card}\left(\left\{n \in\{C 1, C 2\} \mid F_{n} \vee \neg R_{n}\right\}\right) \geq 1 \Rightarrow F_{G 2}=$ True

When a node is not connected to any switch output, 38 it is always required $\left(R_{n}=1\right)$. Else, its requirement ${ }^{386}$ status is obtained from the Moore machine associated 387 to its upstream switch (in $\mathcal{G}_{S}$ ) as explained in the next ${ }^{388}$ section.

The activation status of a node $n$ is computed with 390 Eq. (3):

$$
\left\{\begin{array}{ccc}
\text { if } \Gamma_{\mathcal{G}_{F}}^{-}(n) \neq \varnothing \quad M_{n}=R_{n} \cdot \max _{g \in \Gamma_{\mathcal{G}_{F}}^{-}(n)}\left(M_{g} . v((g, n))\right) & 392 \\
\text { else } & M_{n}=R_{n} & 394 \\
& 395
\end{array}\right.
$$

where $M_{g}$ is the activation status of an upstream gate $g$ and $v((g, n))$ is the label of the edge between $g$ and $n$ in ${ }^{397}$ $\mathcal{G}_{F}$.

For the example of Figure 2, for instance: $M_{C 2}={ }^{399}$ $R_{C 2} \cdot \max \left(M_{G 2}, M_{G 3}\right)$ (the activation status of $C 2$ is ${ }^{400}$ equal to 1 when $G 2$ is activated and 2 when $G 3$ is ac- ${ }^{401}$ tivated.) 402

Each possible value of the activation status of a leaf ${ }^{403}$ refers to a Markov chain of the associated SMP. For ${ }^{404}$ each leaf $l \in L$, while $M_{l}=i(i \in \mathbb{N})$ the active ${ }^{405}$ Markov chain of $\operatorname{smp}(l)$ has to be the chain number $i^{406}$ $\left(M_{l}=i \Longrightarrow X_{l} \in \mathcal{X}_{i}^{\operatorname{smp}(l)}\right)$.

\subsection{Switch behavior modeling}

The role of a switch is to set/reset the requirement ${ }^{410}$ statuses of the nodes that are connected to its outputs 411 according to the values of its inputs and the reconfigura- ${ }^{412}$ tion strategy which is described by the associated Moore ${ }^{413}$ machine.

A Moore machine [19] is an automaton with inputs 415 and outputs which is defined as follows:

Definition 4. A Moore Machine is defined as a 6-tuple ${ }^{417}$ $M=<Q^{M}, Q_{0}^{M}, \Sigma_{I}^{M}, \Sigma_{O}^{M}$, trans $^{M}$, out $^{M}>$ where:

- $Q^{M}$ is a finite set of states;

- $Q_{0}^{M}$ is the initial state;

- $\Sigma_{I}^{M}$ is the input alphabet;

- $\Sigma_{O}^{M}$ is the output alphabet;

- $\operatorname{trans}^{M}: Q^{M} \times \Sigma_{I}^{M} \rightarrow Q^{M}$ is the transition function;

- out ${ }^{M}: Q^{M} \rightarrow \Sigma_{O}^{M}$ is the output function.
In the graphical representation of this automaton (Figure $2 \mathrm{c}$ ), the labels of the transitions are elements of the input alphabet and the elements of the output alphabet are associated to the states.

It is then possible to represent any reconfiguration strategy with a Moore machine by defining the input/output alphabets of this machine as follows, assuming that the elements of the input (output) alphabets are ordered according to the labels of the edges of $\mathcal{G}_{S}$ that are incoming (outgoing) to (from) the switch to which this machine is associated.

- An element of the input alphabet of a Moore machine represents a combination of states of the nodes which are connected to the inputs of the switch whose behavior is described by this machine. In most cases, it is sufficient to know the failure status $F_{n}$ of a node to characterize its state and select the appropriate reconfiguration strategy. More details on the state of the node are needed sometimes, however; in these cases, the state of the node will be characterized by the active state $X_{l}$ of the associated SMP. Hence, when the switch which is associated to the Moore machine owns $i$ inputs, an element of the input alphabet will be a vector with $i$ components that are either failure statuses or SMP states. For the Moore machine $M 1$ at Figure 2 for instance, the elements of the input alphabet are built from the possible states $(W, F)$ of the SMP associated to $C 4$ (first input) and the failure status (True, False) of G2 (second input).

- An element of the output alphabet of a Moore machine represents a combination of requirement statuses of the nodes which are connected to the outputs of the switch whose behavior is described by this machine. For the same example, the elements of the output alphabet are built from the possible requirement statuses $(0,1)$ of $G 2$ and $G 3$.

Globally, this machine describes a reconfiguration strategy where $G 2$ must be required and $G 3$ must not when $G 2$ is faultless (state $q_{0}$ of the Moore machine) and vice versa when $G 2$ is faulty (state $q_{1}$ of the Moore machine). This strategy may fail in case of failure of $C 4$. No state change is possible indeed in this case, even if necessary.

The formula that describes how the requirement status of a node $n$ is updated can now be given:

$$
\left\{\begin{array}{cc}
\text { if } \exists s \in S \mid(s, n) \in E_{S} & R_{n}=\left(o u t^{s t r(s)}\left(U_{S}\right)\right)_{v((s, n))} \\
\text { else } & R_{n}=1
\end{array}\right.
$$


where $U_{s}$ denotes the active state of the Moore ma- 456 chine associated to the switch $s$, and $o u t^{\text {str(r) }}($ is the out- 457 put function of this Moore machine (cf. Definition 4), 458 thus $\left(o u t^{\operatorname{str}(s)}\left(U_{s}\right)\right)_{v((s, n))}$ is the element number $v((s, n))$ of the output of the Moore machine $\operatorname{str}(s)$ when its active state is $U_{s}$. For the example of Figure 2, for instance: $R_{G 3}=\sigma_{1}$ with $\left(\sigma_{0}, \sigma_{1}\right)=o u t^{M 1}\left(U_{S 1}\right)$

Last, it can be noted that the behavior of a BDMP trigger can be modeled (Figure 3 ) by a Moore machine with only one input (the failure status of the origin of the trigger) and one output (the requirement status of the destination of the trigger). Only one strategy is possible however: the destination is required whenever the origin is faulty and not required otherwise. The control of the reconfiguration is obviously out of the scope of this modeling, as pointed out in subsection 2.3.

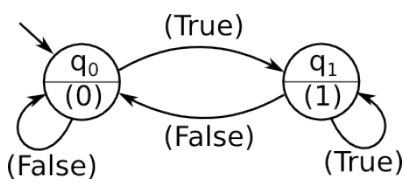

Figure 3: Moore machine that models the strategy of a BDMP trigger

\subsection{Consistency properties}

A GBDMP model is obtained by integrating a representation of the structure of the system by using a fault tree $\mathcal{G}_{F}$ and a graph $\mathcal{G}_{S}$ that describes the inputs and outputs of switches, switched Markov processes to describe the dysfunctional behavior of the leaves of the fault tree and Moore machines to describe the functional behavior of the switches. To ensure consistency of this model, five properties that must be satisfied by any GB- 465 DMP have been defined.

Property 1. The number of sons of a gate must be compatible with its type:

$$
\forall g \in G, \kappa(g) \leq d_{\mathcal{G}_{F}}^{+}(g)
$$

This property means that a $k$-out-of- $n$ gate must have at least $k$ sons.

Property 2. A node (gate or leaf of the fault tree) cannot be connected to several outputs of switches:

$$
\forall n \in N, d_{\mathcal{G}_{S}}^{-}(n) \leq 1
$$

This property avoids conflicts between reconfigura- 478 tion orders.

Properties 3 and 4 focus on switches and their asso- 480 ciated Moore machines. The ranges of the input and 481 output numbers of a switch $s$ will be respectively noted $I^{-}(s)$ and $I^{+}(s)$ :

$I^{-}(s)=\llbracket 0, d_{\mathcal{G}_{S}}^{-}(s)-1 \rrbracket$ and $I^{+}(s)=\llbracket 0, d_{\mathcal{G}_{S}}^{+}(s)-1 \rrbracket$.

Property 3. If a switch owns a inputs and boutputs, these inputs (outputs) must be numbered from 0 to $a-1$ $(0$ to $b-1)$.

$$
\begin{aligned}
& \forall s \in S:\left\{v((n, s)) \mid(n, s) \in E_{S}\right\}=I^{-}(s) \\
& \text { and }\left\{v((n, s)) \mid(s, n) \in E_{S}\right\}=I^{+}(s)
\end{aligned}
$$

Property 4. The input (output) alphabet of the Moore machine that describes the behavior of a switch must be consistent with the inputs (outputs) of this switch:

$\forall s \in S:$

$-\forall \sigma \in \Sigma_{I}^{s t r(s)}: \sigma=\left(\sigma_{i}\right)_{i \in I^{-}(s)} \mid$

$$
\forall i \in I^{-}(s),\left\{\begin{array}{cc}
\text { if In }(s, i) \in C & \sigma_{i} \in\{\text { False,True }\} \\
\text { else } & \vee \sigma_{i} \in \mathcal{X}^{\operatorname{smp}(\operatorname{In}(s, i))} \\
\sigma_{i} \in\{\text { False }, \text { True }\}
\end{array}\right.
$$

- $\forall \sigma \in \Sigma_{O}^{s t r(s)}: \sigma=\left(\sigma_{i}\right)_{i \in I^{+}(s)} \mid \forall i \in I^{+}(s), \sigma_{i} \in\{0,1\}$ where In $(s, i)$ denotes the node $n$ such that: $(n, s) \in E_{S} \wedge v((n, s))=i$.

Finally, a global property of the graph $G$ is stated by Property 5. The role of this property will be discussed at section 4.3.

Property 5. There is no circuit of $\mathcal{G}_{F} \cup \overline{\mathcal{G}_{S}}$ which contains a path of $\mathcal{G}_{F}\left(\overline{\mathcal{G}_{S}}\right.$ designates the graph $\mathcal{G}_{S}$ whose edges have been reversed).

$$
\begin{aligned}
& \forall(x, y) \in N^{2} \text {, there is a path from } x \text { to } y \text { in } \mathcal{G}_{F} \\
& \Longrightarrow \text { there is no circuit through } x \text { and } y \text { in } \mathcal{G}_{F} \cup \overline{\mathcal{G}_{S}} \text {. }
\end{aligned}
$$

Verification of these properties can be done when building the model and is not a real issue because they are static, i.e. they do not depend on the current state of the model.

A GBDMP model that satisfies these properties is called well-formed. Its evolutions in response to sequences of events can be analyzed once the semantics of GBDMP has been formally defined. This is the objective of the next section.

\section{GBDMP semantics}

The global state of a GBDMP at a given date is completely defined by the set of the state variables of every leaf $\left(X_{l} \in \mathcal{X}_{\operatorname{smp}(l)}, \forall l \in L\right)$ and the set of the state variables of every switch $\left(U_{s} \in Q_{s t r(s)}, \forall s \in S\right)$. Hence, the dynamic behavior of a GBDMP can be represented by a state model whose states are global states of the GBDMP and transitions are determined as explained below. 


\subsection{Spontaneous and provoked events}

The evolutions of a GBDMP model are driven by two ${ }^{525}$ types of events:

- spontaneous events: A spontaneous event is an un- 528 controllable event; its occurrence date is a ran- ${ }_{529}$ dom variable. Failure events (except failure ondemand), repair events, phase change events are ${ }^{530}$ examples of spontaneous events. They correspond ${ }^{531}$ to the solid arrows in the SMP representation (cf. ${ }^{532}$ Figure 2 b)).

- provoked events: A provoked event is the conse- 535 quence of a spontaneous event. As the reactions of ${ }_{536}$ the GBDMP are assumed instantaneous, the date of ${ }_{537}$ such an event is the same as that of its cause. Oper- ${ }_{538}$ ation mode changes, e.g. from standby to working, 539 and failure on-demand are examples of provoked 540 events. When several provoked events are concur- 541 rent for a leaf after the occurrence of a given spon- 542 taneous event, the probabilities of those events are 543 given by the transfer function of the correspond- 544 ing SMP. These events correspond to the dashed 545 arrows in the SMP representation (cf. Figure 2 b)). 546

\subsection{GBDMP evolution rules}

The initial state of a GBDMP model is obtained as follows:

1. The active state of every Moore machine is its initial state: $\forall s \in S, U_{s}=q_{0}^{s t r(s)}$.

2. The requirement and activation statuses of every node are computed respectively according to Eqs. (4) and (3).

3. The initial state of every leaf can then be determined using the initial probability distribution $p 0_{M_{l}}^{s m p(l)}$ of the corresponding SMP.

4. The failure status of every node is computed according to Eqs. (1) and (2).

The state of a GBMP is said stable if and only if the ${ }_{551}$ activation and failure status of every leaf complies with ${ }_{552}$ the state of the associated SMP. The stability condition ${ }_{553}$ of a state is formally given at Eq. (5).

$$
\forall l \in L,\left\{\begin{array}{l}
X_{l} \in \mathcal{X}_{M_{l}}^{s m p(l)} \\
\left(F_{l} \wedge X_{l} \in \mathcal{X}_{F}^{s m p(l)}\right) \vee\left(\neg F_{l} \wedge X_{l} \notin \mathcal{X}_{F}^{s m p(l)}\right)
\end{array}\right.
$$

A stable state can change only when a spontaneous 560 event occurs. The state of a leaf is then changed and the ${ }_{561}$ new stable state of the GBDMP is determined by:
1. Updating every other variable (statuses of nodes and active state of Moore machines).

2. If the new state is not stable, provoked events occur to set every SMP in the correct mode. If one of these events is a failure on-demand, steps 1 and 2 must be repeated until the reached state is stable.

It must be noted that the loop introduced above (repetition of the steps 1 and 2) is not infinite because at worst it will finish when every component will be faulty. Computation of the new stable state, which is a fixed point research characterized by the stability condition, always converges.

In response to spontaneous events, a GBDMP model evolves from stable state to stable state by crossing unstable states. This is illustrated at Figure 4, for the example of Figure 2, where solid and dashed rectangles represent respectively stable and unstable states. It is assumed that the probability of the initial state of the SMP associated to every leaf is equal to 1 to define the initial state of the GBDMP. From this state, the evolution starts when the leaf $C 1$ fails what causes the evolution of $S 1$ from $q_{0}$ to $q_{1}$. This evolution implies that $C 1, C 2$ and $C 3$ have to be switched respectively into mode 0,2 and 1 , what explains the following occurences of the three provoked events. The final state is stable according to Eq. (5).

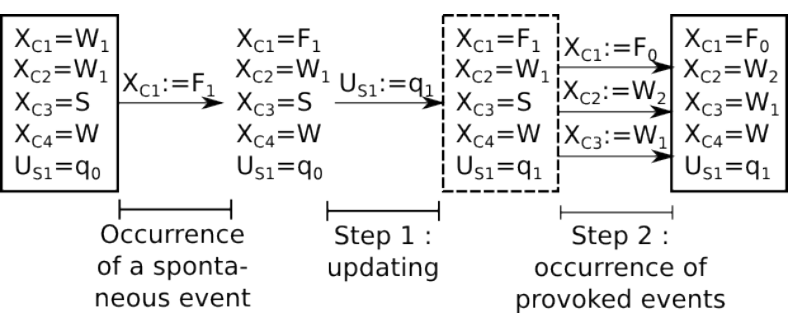

Figure 4: Example of evolutions between two stable states

\subsection{Simulation of a GBDMP}

Once the evolution rules defined, an algorithm to obtain the evolutions of a GBDMP in response to a sequence of spontaneous events has been developed (Algorithm 1). It is assumed that simultaneous occurrences of spontaneous events are not possible. Hence, as an evolution of the GBDMP between two successive stable states is instantaneous (instantaneous reaction of the GBDMP), the GBDMP is always in a stable state when a spontaneous event occurs.

Dependency analysis of the variables which characterize a GBDMP state (statuses and state variables of SMP and Moore machines) must be performed before 
computing their new values because these variables are highly interdependent, as illustrated at figure 5.

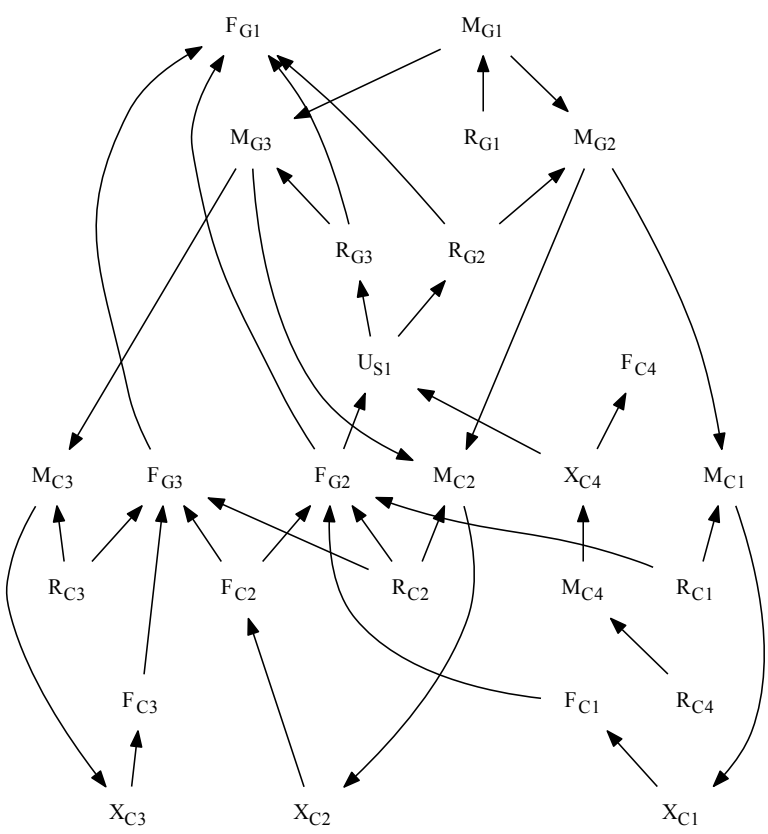

Figure 5: Dependency graph for the variables of Figure 2

It must be underlined that the updating of variables is possible if and only if the GBDMP is well-formed and in particular satisfies property 5 . When this is the case, it is performed in Algorithm 1 by ranking the vertices (nodes and switches) $V$ of the considered GBDMP according to their relative positions in $\mathcal{G}_{F}$ and $\mathcal{G}_{S}$.

A prototype tool named SAGE (Safety Analysis in a GBDMP Environment) has been developed to implement this algorithm. This tool includes also edition and simulation functions and has been used to build and analyze the three examples of the next section.

\section{Examples}

The aim of this section is to show the modeling capabilities of the GBDMP framework on the basis of three simple but representative examples. Several reconfiguration strategies and failure of the control of these strategies are addressed in the first example. The second example focuses on components with more than two operation modes and the third one on a simple phasedmission system. For each example, the corresponding $\overline{\text { Algorithm } 1 \text { Discrete Event Simulation of a GBDMP }}$ model

Require: $\quad<\quad<, E, \kappa, v, s t r, s m p>$ a well-formed GBDMP model (cf. Definitions 2, 3, 4 and Rules 1, 2, 3, 4 and 5).

- $\sigma=\left[e_{1}, \ldots, e_{k}\right]$ a sequence of spontaneous events.

Ensure: A possible evolution of the GBDMP model.

1: \{\}Initialization:

2: $\operatorname{lev}_{\max }:=\max _{v \in V}(\operatorname{Level}(v))$

3: $\forall n \in N: F_{n}:=$ False

4: $\forall s \in S: U_{s}:=q_{0}^{s t r(s)}$

5: lev $:=l e v_{\max }$

while $l e v \geq 0$ do

$\forall n \in N \mid \operatorname{Level}(n)=l$ : to initialize $R_{n}$

$l e v:=l e v-1$

end while

while $l e v \leq l e v_{\max }$ do

$\forall n \in N \mid \operatorname{Level}(n)=l:$ to initialize $M_{n}$

$l e v:=l e v+1$

\section{: end while}

$\forall l \in L:$ to initialize $X_{l}$ using $p 0_{M_{l}}^{\operatorname{smp}(l)}$

: $\}$ Main loop:

$i:=0$

while $i \leq k$ do

\section{if $i \neq 0$ then}

19: $\quad$ occurrence of $e_{i}$ \{\}modification of the state variable for the related leaf.

\section{end if}

is table $:=$ False

while is table $=$ False do

while $l e v \geq 0$ do

$\forall n \in N \mid \operatorname{Level}(n)=l$ : to update $F_{n}$

$\forall s \in S \mid \operatorname{Level}(s)=l$ : to update $U_{s}$

$\forall n \in N \mid \operatorname{Level}(n)=l$ : to update $R_{n}$

$l e v:=l e v-1$

\section{end while}

while $l e v \leq l e v_{\max }$ do

$\forall n \in N \mid \operatorname{Level}(n)=l$ : to update $M_{n}$ $l e v:=l e v+1$

end while

$\forall l \in L \mid X_{l} \notin \mathcal{X}_{M_{l}}^{s m p(l)}$ : to update $X_{l}$ \{\}occurrence of provoked events

34: if $\forall l \quad \in \quad L \mid\left(F_{l} \wedge X_{l} \in \mathcal{X}_{F}^{\operatorname{smp}(l)}\right) \quad \vee$ $\left(\neg F_{l} \wedge X_{l} \notin \mathcal{X}_{F}^{s m p(l)}\right)$ then

is S table $:=$ True

end if

end while

$i:=i+1$

end while 
GBDMP model is detailed and the evolution of this 621 model in response to a sequence of failure and repair 622 events is analyzed; for simplicity reasons, it will be assumed in these analyses that the probability of the initial ${ }_{223}$ state of the SMP associated to every leaf is equal to $1{ }^{624}$ and that every transition between the Markov chains of 625 this SMP is deterministic.

\subsection{Two different reconfiguration strategies imple mented on control devices that may fail}

\subsubsection{Example description}

This example (Figure 6) comprises two groups of redundant components $(C 1 a, C 1 b, C 1 c)$ and $(C 2 a, C 2 b)$; the nature of these components does not matter. Every component can be in active mode or standby mode and may fail and be repaired in both modes.

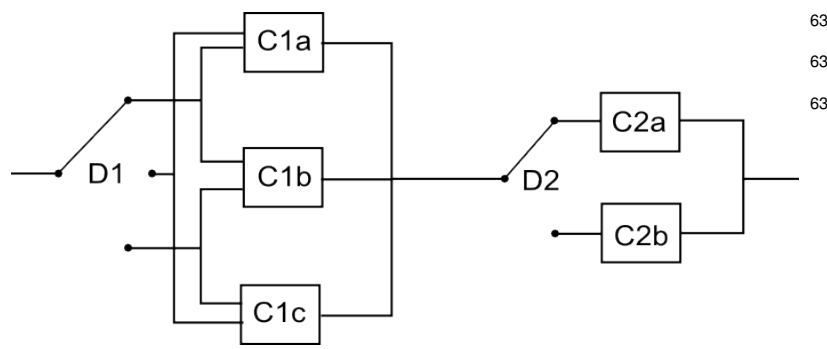

Figure 6: Two groups of components with different reconfiguration strategies

The strategies selected for the two groups are different, however:

- The first group performs correctly its service when at least two components among the three ones are faultless; by default, $C 1 a$ and $C 1 b$ are active and $C 1 c$ in standby. When one of the active components fails, it is replaced by the standby component if it is faultless. The operation of the failed component is resumed only when it is repaired and one of the currently active components fails. This type of resumption of operation for a repaired component ${ }^{638}$ will be termed resuming at the latest.

- The second group performs correctly its service when at least one component among the two ones is faultless; $C 2 a$ must be active whenever it is faultless. Hence, when this component is repaired after it has failed, it must immediatly be set in its active mode. This type of resumption will be termed resuming at the earliest.

The two strategies will be modeled by different Moore machines that will be described in what follows. ${ }_{643}$
Furthermore, the control devices $D 1$ and $D 2$ own two failure modes:

- frozen (the output of the device is stuck in its current position and the combination of active components cannot be modified); the failure and repair rates are respectively $\lambda_{f}$ and $\mu_{f}$.

- bad contact (the output of the device is in open circuit and no combination of active components can be selected); the failure and repair rates are respectively $\left(\lambda_{b c}\right.$ and $\left.\mu_{b c}\right)$.

\subsubsection{Modeling}

The GBDMP representation of the structure of the example is given at figure 7 . In addition to the classical fault tree, this well-formed model includes two switches and two leaves that correspond to the devices where the control of the reconfiguration is implemented ( $D 1$ and D2).

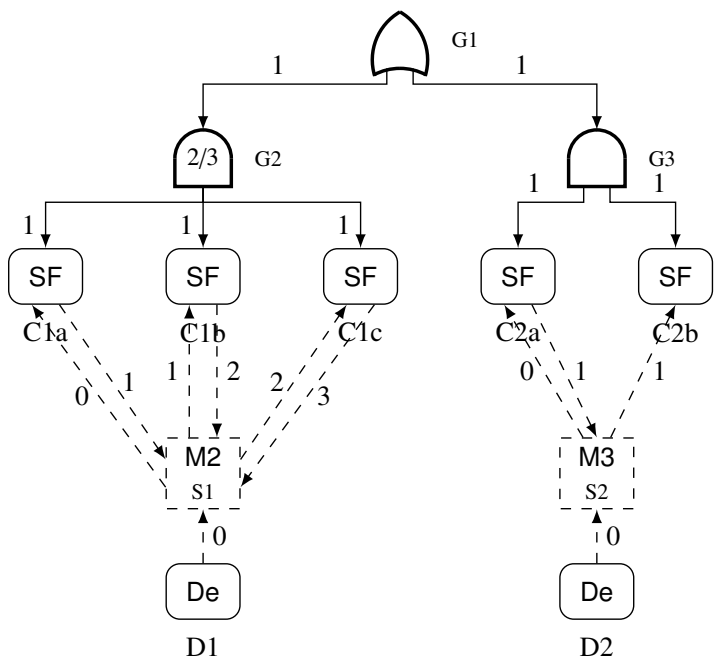

Figure 7: Model of the structure of the example of Figure 6

The SMP associated to the leaves $C 1 a$ to $C 2 b$ is a classical 2-SMP and that associated to $D 1$ and $D 2$ is a 1-SMP that is shown at figure 8.

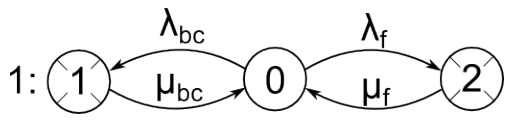

Figure 8: Switched Markov Process De

A first benefit of the GBDMP framework must be clearly highlighted at this point. Using SMP to describe the behavior of the leaves permits to consider several 
failure modes, even for the control of the reconfigura- 672 tion.

Last, the Moore machines $M 2$ and $M 3$ that describe respectively the behavior of the GBDMP switches $S 1$ and $S 2$ are given at figure 9 . These machines model the two reconfiguration strategies previously described. The elements of the input alphabet of $M 2(M 3)$ are combinations of the active state of the SMP of $D 1(D 2)$ and the failure statuses of $C 1 a, C 1 b$ and $C 1 c(C 2 a, C 2 b)$; the elements of the output alphabet are combinations of the requirement statuses of $C 1 a, C 1 b$ and $C 1 c(C 2 a$, $C 2 b$ ). The character - means that any value is possible. For $M 2$ for instance, $\left(2,{ }_{-}\right.$, _ $\left._{-}\right)$means that $D 1$ is faulty, the failure mode being frozen, and $C 1 a, C 1 b$ and $C 1 c$ can be faulty or not; the operation mode of $C 1 a, C 1 b$ and $C 1 c$ cannot be modified in this case, whatever it should be. $(0, T, F, F)$ means that $D 1$ is faultless, $C 1 a$ faulty, $C 1 b$ and $C 1 c$ faultless; $C 1 a$ is no more required and must be replaced by $C 1 c$ (transition from $q_{0}$ to $q_{2}$ ).

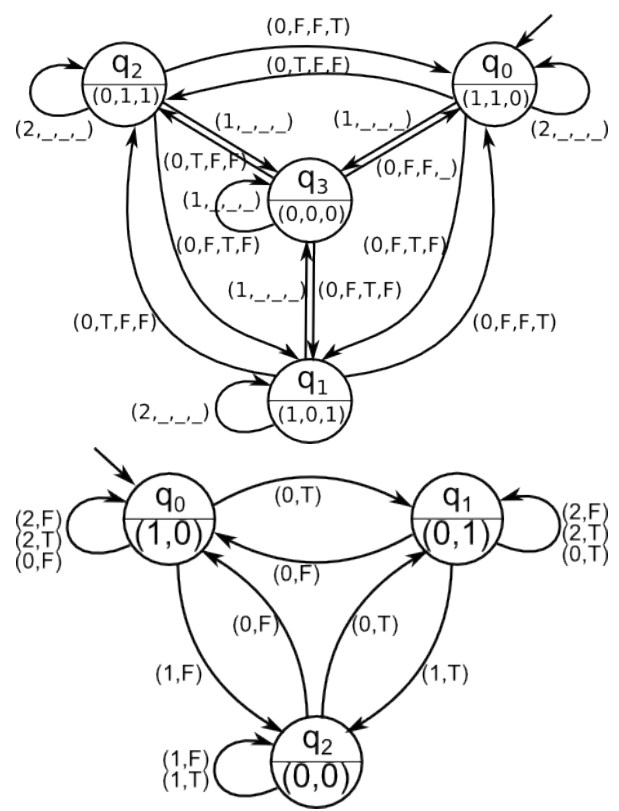

Figure 9: Moore machine $M 2$ (on the top) and $M 3$ (at the bottom) mode once repaired (resuming at the latest strategy).

Table 1: Example of evolution for the first group ( $S:$ Standby, $F 1$ : Faulty and standby, $W$ : Working and $F 2$ : Faulty and working)

\begin{tabular}{|c|c|c|c|c|c|}
\hline sequence & \multicolumn{5}{|c|}{$0 \stackrel{f_{C l c}}{\longrightarrow} 1 \stackrel{f_{C l a}}{\longrightarrow} 2 \stackrel{r_{C l c}}{\longrightarrow} 3 \stackrel{r_{C l a}}{\longrightarrow} 4$} \\
\hline$X_{C 1 a}$ & $W$ & $W$ & $F_{2}$ & $F_{1}$ & $S$ \\
\hline$X_{C 1 b}$ & $W$ & $W$ & $W$ & $W$ & $W$ \\
\hline$X_{C 1 c}$ & $S$ & $F_{1}$ & $F_{1}$ & $W$ & $W$ \\
\hline$F_{G 1}$ & False & False & True & False & Fals \\
\hline
\end{tabular}

The results of a similar analysis with the tool SAGE for the second group of components is given at Table 2. The sequence is $f_{C 2 a} \rightarrow f_{D 2}^{f r o z e n} \rightarrow r_{C 2 a} \rightarrow f_{C 2 b}$, where $f_{C 2 a}\left(f_{C 2 b}\right)$ represents the failure of $C 2 a(C 2 b), r_{C 2 a}$ the repairs of $C 2 a$, and $f_{D 2}^{f r o z e n}$ the failure of $D 2$ in the frozen mode. The states of $C 2 a, C 2 b, D 2$ and the failure status of $G 2$ are given in the rows of this table.

This analysis highlights strongly the interest of the GBDMP framework, where failures of the control are considered, for MBSA. When $C 2 b$ fails indeed, the service is no more provided ( $G 2$ becomes faulty) while $C 2 a$ has been previously repaired because $D 2$ is faulty, in a frozen failure mode; the control of the reconfiguration is lost. This significant result could not be obtained with other frameworks that do not consider control devices failures.

Table 2: Example of evolution for the second group ( $S$ : Standby, $F 1$ : Faulty and standby, $W$ : Working and $F 2$ : Faulty and working)

\begin{tabular}{|c|c|c|c|c|c|}
\hline sequence & \multicolumn{5}{|c|}{$0 \stackrel{f_{C 2 a} a}{\longrightarrow} 1 \stackrel{f_{D 2}^{f^{\prime r z a n}}}{\longrightarrow} 2 \stackrel{r_{C 2 a}}{\longrightarrow} 3 \stackrel{f_{C 2 b}}{\longrightarrow} 4$} \\
\hline$X_{C 2 a}$ & \begin{tabular}{l|}
$W$ \\
\end{tabular} & $F_{1}$ & $F_{1}$ & $S$ & $S$ \\
\hline$X_{C 2 b}$ & $S$ & $W$ & $W$ & $W$ & $F_{2}$ \\
\hline$X_{D 2}$ & 0 & 0 & 2 & 2 & 2 \\
\hline$F_{G 1}$ & False & False & False & False & True \\
\hline
\end{tabular}

\subsection{Multi-state pumps}

\subsubsection{Example description}

Some industrial plants comprise pumps which own 3 operation modes:

- $O f f$. The pump is inactive (in standby mode). It cannot fail but can be repaired with a repair rate $\mu$.

- On. The pump is in its normal operation mode. It can fail with a failure rate $\lambda$ and be repaired with a repair rate $\mu$.

- Over. The pump is in an overspeed operation mode. It can fail with a greater failure rate $4 \lambda$ and be repaired with the same repair rate $\mu$. 
An example of use of two such pumps is shown at figure 10. The service is correctly performed when either both pumps are working in normal operation mode or one pump is in Over mode; the latter solution is selected when one pump has failed.

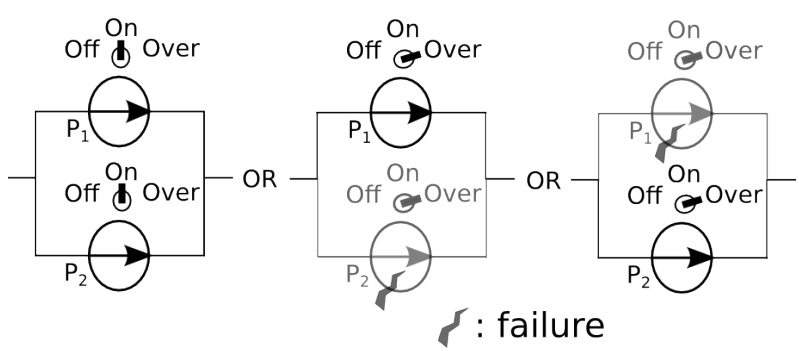

Figure 10: Two pumps with different operation modes

\subsubsection{Modeling}

The GBDMP of this example is given at Figure 11. In the structure view (Figure 11 a), the edges which connect both leaves to gates $G 2$ and $G 3$ have dierent labels because $G 2$ fails when at least one pump fails in normal operation mode, while $G 3$ fails when both pumps have failed in the Over mode indeed. With other words, when $G 2$ is required and not $G 3, P 1$ and $P 2$ are in mode $O n$, whereas when $G 3$ is required (whatever the value of the requirement status of $G 2$ ) they are in mode Over. Indeed, according to Eq. (3):

- $R_{G 2}=1 \wedge R_{G 3}=0 \Rightarrow M_{G 2}=1 \wedge M_{G 3}=0 \Rightarrow$ $M_{P 1}=M_{P 2}=\max \left(1 . M_{G 2}, 2 \cdot M_{G 3}\right)=1$

- $R_{G 2}=1 \wedge R_{G 3}=1 \Rightarrow M_{G 2}=1 \wedge M_{G 3}=1 \Rightarrow$ $M_{P 1}=M_{P 2}=\max \left(1 . M_{G 2}, 2 \cdot M_{G 3}\right)=2$

In the 3-SMP $P u$ associated to every leaf (Figure $11 \mathrm{~b}$ ), the three chains 0,1 and 2 correspond to respectively the ${ }_{744}$ operation modes Off, On and Over. The only element of ${ }_{745}$ the input (output) alphabet of the Moore machine (Fig- 746 ure $11 \mathrm{c}$ ) is the failure status of $G 2$ (requirement status 747 of $G 3$ ); therefore, the role of the switch is to require $G 3 \quad 748$ when $G 2$ has failed.

\subsubsection{Simulation}

Table 3 shows the results of Algorithm 1 for the se- 752 quence $f_{P 1} \rightarrow f_{P 2} \rightarrow r_{P 1} \rightarrow r_{P 2}$, with the same no- 753 tations than for the first example. These results corre- 754 spond to the expected behavior; when one pump fails, 755 the operation mode of the remaining faultless pump is 756 switched to the Over mode and when both pumps are 757 faultless their operation mode is $\mathrm{On}$. This example 758 shows that multi-state components with more than two 759

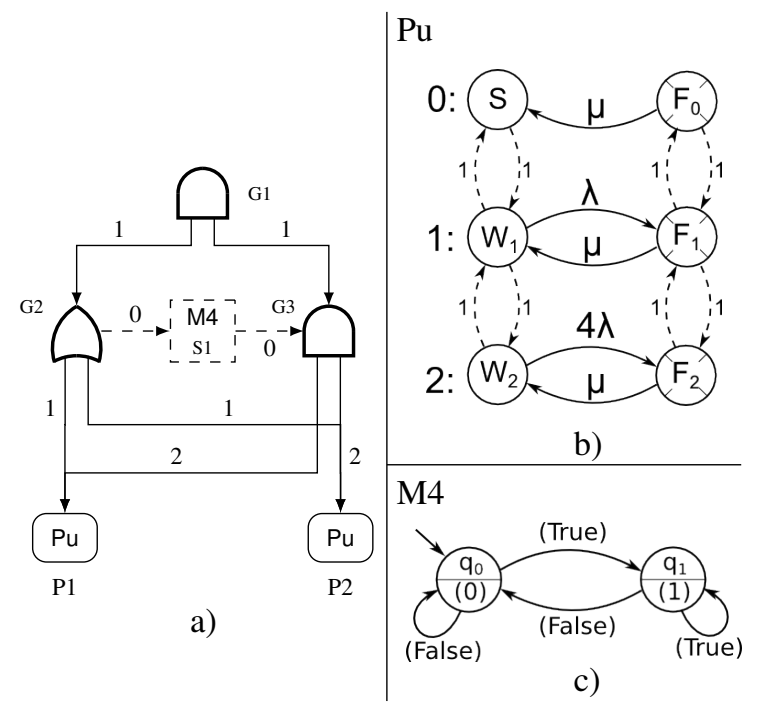

Figure 11: GBDMP model for the example of Figure 10

operation modes can easily be considered into a GBDMP model.

Table 3: Behavior of the model for a scenario that involves $P 1$ and $P 2$

\begin{tabular}{|c|c|c|c|c|l|}
\hline sequence & \multicolumn{4}{|c|}{$0 \stackrel{f_{P 1}}{\longrightarrow} 1 \stackrel{f_{P 2}}{\longrightarrow} 2 \stackrel{r_{P 1}}{\longrightarrow} 3 \stackrel{r_{P 2}}{\longrightarrow} 4$} \\
\hline$X_{P 1}$ & $W_{1}$ & $F_{2}$ & $F_{2}$ & $W_{2}$ & $W_{1}$ \\
\hline$X_{P 2}$ & $W_{1}$ & $W_{2}$ & $F_{2}$ & $F_{2}$ & $W_{1}$ \\
\hline$F_{G 1}$ & False & False & True & False & False \\
\hline
\end{tabular}

\subsection{A simple phased mission system}

\subsubsection{Example description}

A simple plant where two liquids are poured in a tank then mixed is sketched at Figure 12. In the first phase, the valve $V 1$ is open and the valve $V 2$ closed to pour the first liquid; when the phase change event $\delta_{12}$ occurs, the valve $V 1$ is closed and the valve V2 opened to pour the second liquid and so on. Both valves may fail stuckopen or stuck-closed. Every failure is revealed only when the operation mode of the valve must be changed for a phase change and may be considered as a failure on-demand. It will be assumed that the failure (repair) rate $\lambda(\mu)$ is the same for the two types of failure.

This plant may be seen as a very simple phasedmission system. Despite its structure remains unchanged, the dysfunctional behavior and success criterion of its components change from one phase to the other one indeed. When $V 2$ is stuck-open for instance, this valve is faulty during the first phase and faultless in the second one. 

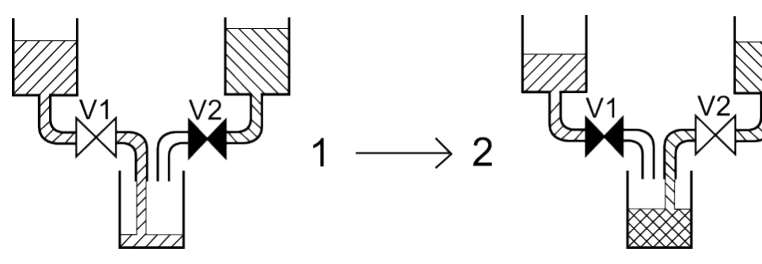

Figure 12: Two valves performing a mixture in two phases

\subsubsection{Modeling}

The dysfunctional behavior of a valve is modeled by the 2-mode SMP Va (Figure $13 \mathrm{~b}$ ). The two Markov chains 0 and 1 represent respectively the dysfunctional behavior when the valve is expected closed and open. When the active state of this SMP for $V 2$ is $C$ for instance, it can evolve to $O$ for a phase change (phase 1 to phase 2), provided that $V 2$ be faultless, or to ?SC if $V 2$ fails during phase 1 . This failure will be detected only at the phase change (transition from ?SC to $S C_{1}$ ).

In the structure view (Figure 13 a), the edges which connect both leaves $V 1$ and $V 2$ to gates $G 2$ and $G 3$ have 785 different labels because when $G 2(G 3)$ is active, the first 786 (second) phase is performed; hence $V 1(V 2)$ is expected 787 to be open and $V 2(V 1)$ closed.

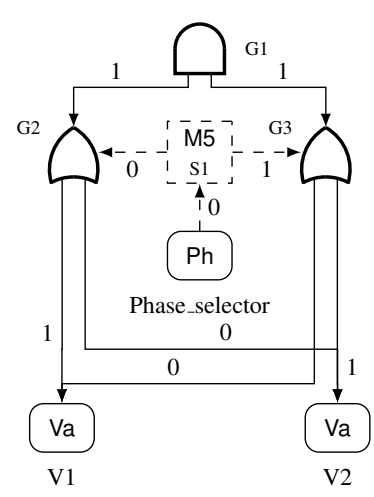

a)

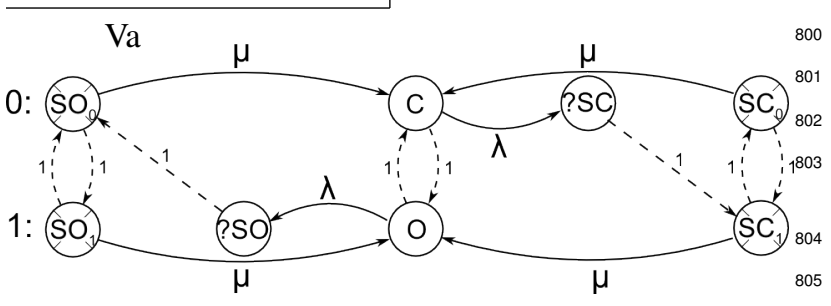

Figure 13: GBDMP model for the example of Figure $12^{4}$

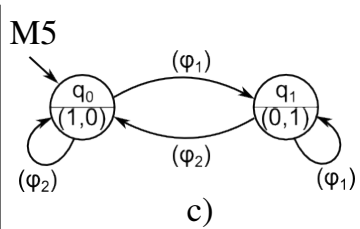

$\mathrm{Ph}$

\section{1:}

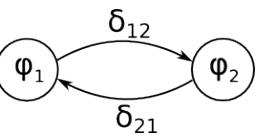

b)

\section{5}

806
${ }^{4}$ In SMP $P h: \varphi_{i}$ means phase $i$ is the active phase. In SMP Va: $C=$ Closed; ?SC = undetected Stuck-Closed; $S C_{0}=$

\subsubsection{Simulation}

Table 4 shows the results of algorithm 1 for sequence stuck $_{V 1} \rightarrow \delta_{12} \rightarrow r_{V 1}$ where stuck $k_{V 1}$ represents a stuckopen failure of $V 1$ during the first phase (transition from $O$ to ?S $O$ in the SMP of $V 1)$ and $r_{V 1}$ the repairs of this failure (transition from $S O 0$ to $C$ in the SMP of $V 1$ ). The results correspond to what was forecast, e.g. $G 1$ becomes faultless only when $V 1$ has been repaired even if the state of $V 2$ has correctly changed after the occurrence of $\delta 12$.

Table 4: Behavior of the model for a scenario that involves $V 1$ and the phase selector

\begin{tabular}{|c|c|c|c|l|}
\hline sequence & $0 \stackrel{\text { stuck }}{\longrightarrow} 1 \stackrel{\delta_{12}}{\longrightarrow} 2 \stackrel{r_{V 1}}{\longrightarrow} 3$ \\
\hline$X_{V 1}$ & $O$ & $? S O$ & $S O_{0}$ & $C$ \\
\hline$X_{V 2}$ & $C$ & $C$ & $O$ & $O$ \\
\hline$X_{\text {Phase_select }}$ & $\varphi_{1}$ & $\varphi_{1}$ & $\varphi_{2}$ & $\varphi_{2}$ \\
\hline$F_{G 1}$ & False & False & True & False \\
\hline
\end{tabular}

This example has showed that components with several failure models can be modeled in the GBDMP framework and that mission-phased systems can be considered too, what is not surprising because phase change is a particular reconfiguration mechanism.

\section{Qualitative and quantitative analysis}

This section aims to show how the choice of a reconfiguration strategy impacts the results of qualitative and quantitative analysis. To meet this objective, only one basic example, a classical standby redundancy system with two components $\mathrm{A}$ and $\mathrm{B}$, will be focused on. It will be assumed that every component may fail on demand; hence, its dysfunctional behavior is depicted by the SMP of Figure 15. This model is easily obtained from that of Figure $1 \mathrm{~b}$ ) by adding a transition from the state $S$ (Standby) to the state $F 2$ (Faulty during working); $\gamma$ is the failure on demand rate.

Four GBDMP models of the considered system are proposed at Figure 14:

1. The reconfiguration strategy of the first model is identical to that of a BDMP trigger and no failure of this strategy is considered. Hence, this model behaves strictly as a BDMP.

Stuck-Closed, expected closed; $S C_{1}=$ Stuck-Closed, expected open; $O=$ Open; ? $S O=$ undetected Stuck-Open; $S O_{0}=$ Stuck-Open, expected closed; $S O_{1}=$ Stuck-Open, expected open. 


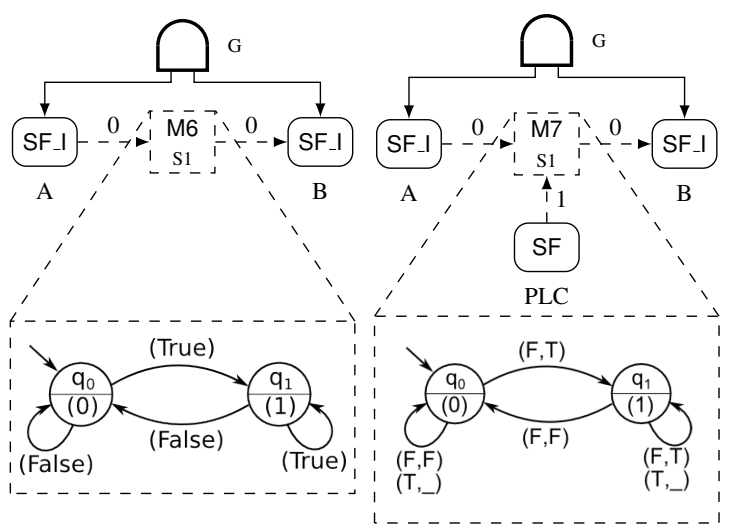

Failure of $A \Longrightarrow$
activation of $B$
Repair of $A \Longrightarrow$
deactivation of $B$

model 1

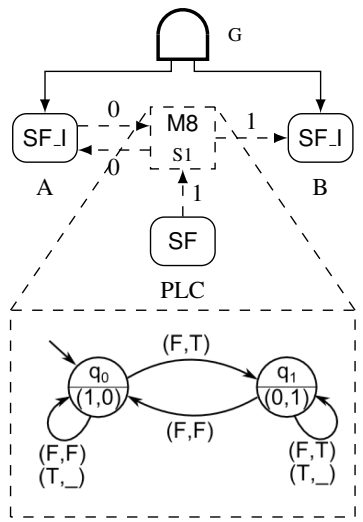

If PLC is not faulty:

Failure of $A \Longrightarrow$

deactivation of $A$, activation of $B$

Repair of $A \Longrightarrow$

activation of $A$,

deactivation of $B$

model 3

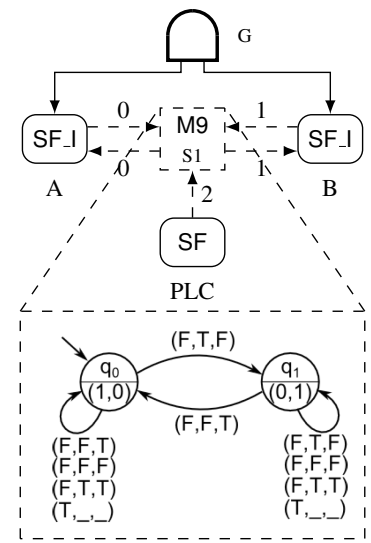

If PLC is not faulty:

Active comp. is faulty and

inactive comp. is not

$\Longrightarrow$

deactivation of active comp. activation of inactive comp.

model 4

Figure 14: Four different reconfiguration strategies to manage a standby redundancy

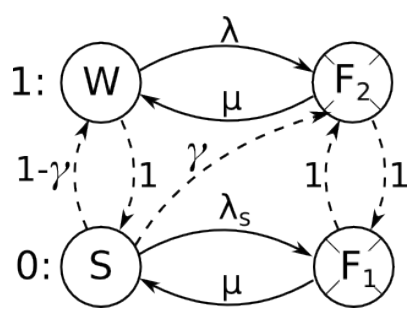

Figure 15: SMP of kind $S F_{-} I$ : model of a component that may fail on 829 demand

2. Failure of the reconfiguration strategy is integrated in the second model. It is assumed that reconfiguration is controlled by a PLC (Programmable Logic Controller) that may fail.

3. Deactivation/activation of component A after failure/repair is explicitly modeled in the third model while this component remained always active in the previous model.

4. Last, the fourth model uses the reconfiguration strategy resuming at the latest that has been defined at subsection 5.1 (the Moore machine M9 is the adaptation for two components of M2, depicted at Figure 9) while the strategy resuming at the earliest was selected for the third model.

These four reconfiguration strategies are formally de-

\footnotetext{
${ }^{5}$ In this table, $f_{A}, f_{B}$ and $f_{P L C}$ means respectively failure of $A, B$ and $P L C$ (either in active mode or in standby mode), and $f !_{B}$ means on demand failure of $B$
} 
Table 5: Minimal Cut Sequences for the four models

\begin{tabular}{|c|c|}
\hline MCS & concerned model \\
\hline$f_{A} f_{B}$ & \\
$f_{A} f !_{B}$ & $1,2,3,4$ \\
$f_{B} f_{A}$ & \\
\hline$f_{P L C} f_{A}$ & $2,3,4$ \\
\hline$f_{A} f_{P L C} r_{A} f_{B}$ & 3,4 \\
\hline$f_{A} r_{A} f_{P L C} f_{B}$ & 4 \\
\hline
\end{tabular}

is not really surprising but motivates an accurate modeling of reconfiguration strategies to forecast relevant set of MCS. The first three MCS are obtained with the four models and easy to interpret: the system fails totally when both components A and B have failed (B may have failed in active or standby mode or on demand). The fourth MCS is also easy understandable: the system fails when A fails after the PLC has failed because the service cannot be then transferred to B. The fifth and sixth MCS require a deeper reasoning because they are longer and include a repair event. For the fifth MCS, the system fails when B fails (last event of the sequence) while A has been previously repaired (third event of the sequence) because the PLC is faulty (second event) and therefore is not able to resume the service from B to A. A similar reasoning can be made for the sixth sequence. Comparison of the models 3 and 4 on the basis of this only analysis leads to favor the strategy resuming at the earliest (strategy selected for the model 3) because the sixth MCS is not possible with this strategy (the service is immediately switched to A once repaired). Nevertheless, this partial conclusion must be smoothed by the results of quantitative analysis of these models.

\subsection{Quantitative analysis}

This analysis will focus on the unavailability of the 900 four models. Several contributions for scalable quanti- 901 tative analysis techniques have been already published 902 (see [22] and [23]). The curves of Figure 16 have 903 been obtained by using the method described in [23] 904 with the following numerical values: $\lambda=10^{-3} h^{-1}$; 905 $\lambda_{S}=5.10^{-4} h^{-1} ; \mu=10^{-1} h^{-1}$ and $\gamma=0.2$ (arbitrary 906 values selected to accentuate the differences). In this 907 approach, which was developed to increase scalability 908 of quantitative analysis, a reduced-size Markov chain 909 which includes only the most likely states is built from 910 a high-level model (such as a GBDMP). Construction 911 of this chain relies on [24] and a state relevance factor ${ }_{912}$ which represents the likelihood of a state and is com- ${ }_{913}$ puted from the transition rates of the SMP.

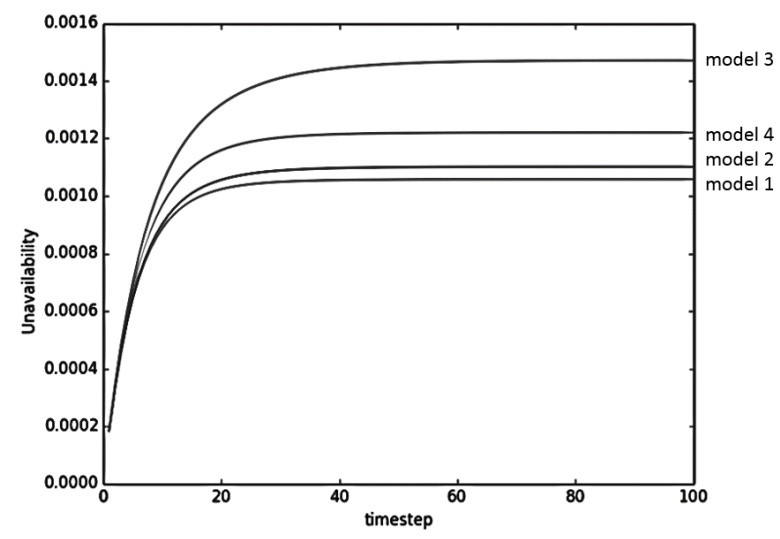

Figure 16: Unavailability for the four models

The unavailability of model 1 is the lowest one simply because the failure of the component that controls the reconfiguration (PLC) is not taken into account in this model. This model is too optimistic. The second model is less unavailable than the third and fourth ones because $\mathrm{A}$ is always active in this model and hence cannot fail on demand. Comparison of the models 3 and 4 leads this time to favor the strategy resuming at the latest (strategy selected for the model 4) because it minimizes the reconfiguration occurrences and consequently the risk of failure on demand. Hence, selecting a resuming strategy requires an expert decision on the bases of both qualitative and quantitative analyses.

\section{Conclusions and Perspectives}

This paper has presented the syntax and semantics of the GBDMP framework that has been developed to allow modeling explicitly and accurately reconfiguration strategies and considering the failures of the control of the reconfiguration. In our opinion, the main novelty of this framework is modeling of the reconfiguration strategies by Moore machines whose inputs depend on failure states of components of the process and the control. To ensure consistency of a GBDMP model that integrates a model of the structure of the system, in the form of fault tree enriched with switches, models of components, in the form of SMP, and switches whose behavior is described by Moore machines, five properties that must be satisfied by a well-formed model have been stated. An algorithm to analyze the evolutions of a GBDMP model in response to a sequence of spontaneous events has been developed and implemented in a prototype tool, too. 
The treatment of representative examples has shown 966 the benefits of this framework: different reconfigura- ${ }^{967}$ tion strategies can be precisely considered, the impact of ${ }^{968}$ failures of the control can be studied, components with ${ }_{970}^{969}$ several operation and failure modes can be introduced. 971 Hence, the initial objective of this work has been met. ${ }^{972}$ Moreover, it has been pinpointed that extension to mod- ${ }^{973}$ eling and analysis of phased-mission systems is possi- 975 ble because phase change is a particular reconfiguration 976 mechanism.

Nevertheless, construction of a GBDMP model is a ${ }^{978}$ difficult task that requires a lot of expertise. To over- 980 come this issue, two solutions are possible. The first ${ }^{981}$ one consists in building libraries of SMP and Moore ${ }^{982}$ machines for typical components and reconfiguration ${ }_{984}^{983}$ strategies to allow modular construction by instantia- 985 tion and assembly. The second one is a posteriori for- ${ }^{986}$ mal verification of dynamic properties of GBDMP mod- ${ }^{987}$ els; model-checking of GBDMP models using the tool ${ }_{989}^{988}$ NuSMV is an on-going work in our laboratory.

\section{Acknowledgment}

This work has been funded by the French Investment ${ }^{996}$ of Future Program: Generic Components of Embedded 998 Software as part of the CONNEXION project.

\section{References}

[1] J.-B. Dugan, S.-J. Bavuso, M.-A. Boyd, Dynamic fault-tree models for fault-tolerant computer systems, IEEE Transactions on Reliability 41 (3) (1992) 363-377.

[2] M. Walker, Y. Papadopoulos, Qualitative temporal analysis: To- ${ }^{1008}$ wards a full implementation of the Fault Tree Handbook, Con- ${ }^{1009}$ trol Engineering Practice 17 (10) (2009) pp. 1115-1125.

[3] A. Bobbio, D.-C. Raiteri, Parametric Fault Trees with dynamic 101 gates and repair boxes, in: Proc. Reliability and Maintainabil- 1012 ity Symposium (RAMS'2004), Los Angeles (California - USA), ${ }^{1013}$ January 2004, pp. 459-465.

[4] J.-B. Dugan, S.-J. Bavuso, M.-A. Boyd, Fault trees and Markov ${ }^{1015}$ models for reliability analysis of fault-tolerant digital systems, ${ }^{1016}$ Reliability Engineering \& System Safety 39 (3) (1993) 291- 1017 307.

[5] G. Merle, J.-M. Roussel, J.-J. Lesage, Algebraic Determination ${ }^{1019}$ of the Structure Function of Dynamic Fault Trees, Reliability ${ }^{1020}$ Engineering \& System Safety 96 (2) (2011) 267-277.

[6] G. Merle, J.-M. Roussel, J.-J. Lesage, Quantitative analysis of ${ }^{1022}$ dynamic fault trees based on the structure function, Quality and ${ }^{1023}$ Reliability Engineering International 30 (1) (2014) 143-156. ${ }_{1024}$

[7] A. Rauzy, Towards a sound semantics for Dynamic Fault Trees, ${ }^{1025}$ Reliability Engineering \& System Safety 142 (2015) 184-191.

[8] D. Ge, M. Lin, Y. Yang, R. Zhang, Q. Chou, Quantitative analysis of dynamic fault trees using improved Sequential Binary Decision Diagrams, Reliability Engineering \& System Safety 142 (2015) 289-299.
[9] D. Ge, D. Li, Q. Chou, R. Zhang, Y. Yang, Quantification of highly coupled dynamic fault tree using IRVPM and SBDD, Quality and Reliability Engineering International 32 (1) (2014) 139-151.

[10] P. Zhu, J. Han, L. Liu, F. Lombardi, A stochastic approach for the analysis of dynamic fault trees with spare gates under probabilistic common cause failures, IEEE Transactions on Reliability 64 (3) (2015) 878-892.

[11] M. Bouissou, J.-L. Bon, A new formalism that combines advantages of fault trees and Markov models: Boolean logic Driven Markov Processes., Reliability Engineering \& System Safety 82 (2) (2003) 149-163.

[12] M. Batteux, T. Prosvirnova, A. Rauzy, L. Kloul, The altarica 3.0 project for model-based safety assessment, in: Proc. $11^{\text {th }}$ IEEE International Conference on Industrial Informatics (INDIN'2013), Bochum (Germany), July 2013, pp. 741-746.

[13] M. Gudemann, F. Ortmeier, A Framework for Qualitative and Quantitative Formal Model-Based Safety Analysis, in: Proc. IEEE 12th International Symposium on High-Assurance Systems Engineering (HASE 2010), San Jose (California - USA), November 2010, pp. 132-141.

[14] M. Lipaczewski, F. Ortmeier, T. Prosvirnova, A. Rauzy, S. Struck, Comparison of modeling formalisms for safety analyses: SAML and AltaRica, Reliability Engineering \& System Safety 140 (2015) 191-199.

[15] P.-Y. Piriou, J.-M. Faure, J.-J. Lesage, Control-in-the-loop Model Based Safety Analysis, in: Proc. $24^{\text {th }}$ European Safety \& Reliability Conference (ESREL'14), Wroclaw (Poland), September 2014, pp. 655-662.

[16] G. Levitin, S. V. Amari, Multi-state systems with multi-fault coverage, Reliability Engineering \& System Safety 93 (11) (2008) 1730c-1739.

[17] R. Peng, H. Mo, M. Xie, G. Levitin, Optimal structure of multistate systems with multi-fault coverage, Reliability Engineering \& System Safety 119 (2013) 18-25.

[18] P.-Y. Piriou, J.-M. Faure, J.-J. Lesage, Modeling standby redundancies in repairable systems as guarded preemption mechanisms, in: Proc. $5^{\text {th }}$ International Workshop on Dependable Control of Discrete Systems (DCDS 2015), Cancun (Mexico), May 2015, pp. 147-153.

[19] E.-F. Moore, Gedanken-experiments on sequential machines, Annals of Mathematical Studies 34 (1956) 129-153.

[20] P.-Y. Chaux, J.-M. Roussel, J.-J. Lesage, G. Deleuze, M. Bouissou, Towards an unified definition of minimal cut sequences, in: Proc. $4^{\text {th }}$ International Workshop on Dependable Control of Discrete Systems (DCDS 2013), Vol. 4 (1), York (UK), September 2013, 6 pages.

[21] P.-Y. Piriou, J.-M. Faure, J.-J. Lesage, A formal definition of minimal cut sequences for dynamic, repairable and reconfigurable systems, in: Proc. $25^{\text {th }}$ European Safety \& Reliability Conference (ESREL'16), Glasgow (UK), September 2016, 8 pages.

[22] O. Yevkin, An efficient approximate markov chain method in dynamic fault tree analysis, Quality and Reliability Engineering International 32 (4) (2015) 1509-1520.

[23] P.-A. Brameret, A. Rauzy, J.-M. Roussel, Automated generation of partial Markov chain from high level descriptions, Reliability Engineering \& System Safety 139 (2015) 179-187.

[24] E.-W. Dijkstra, A note on two problems in connexion with graphs, Numerische mathematik 1 (1) (1959) 269-271. 